\title{
Reducing Departure Delays for Adjacent Center Airports using Time- Based Flow Management Scheduler: Checkbox ON or OFF?
}

\author{
B. Parke, C. Mohlenbrink, C. Brasil, C. Speridakos, \\ H.S. Yoo, F. Omar, N. Buckley, C. Gabriel, A. \\ Belfield, SJSURF/NASA Ames, Moffett Field, CA \\ bonny.parke@nasa.gov
}

\author{
P.U. Lee, N.M. Smith \\ NASA Ames, Moffett Field, CA U.S.
}

\begin{abstract}
Keywords-Airborne delay, departure delay, ground delay, Time-Based Flow Management, TBFM Checkbox

Abstract-The Federal Aviation Administration (FAA) has enhanced the Time-Based Flow Management (TBFM) scheduling tool with a "Checkbox ON" vs. "OFF" function which allows Traffic Management Coordinators (TMCs) to make room in a crowded arrival stream for a departure. When scheduling a departure, having the checkbox ON can delay the Scheduled Times of Arrivals (STAs) of the airborne flights upstream of the TBFM freeze horizons and can compress these flights to their minimum required spacing, thereby creating a slot for a departure. Hence, having the checkbox $O N$ can reduce the frequent ground delays of aircraft departing near high volume airports but can increase delays for airborne arrivals. A Humanin-the-Loop (HITL) simulation compared arrival and departure delays to Newark Airport (EWR) with the checkbox ON vs. OFF as the default position.
\end{abstract}

Three other conditions in this HITL involved various National Airspace System (NAS)-wide approaches for timely delivery of aircraft to the TBFM region. These conditions were: Baseline, using current Mile-in-Trail (MIT) spacing restrictions; Integrated Demand Management (IDM), where all aircraft were given departure times (Expect Departure Clearance Times, or EDCTs), ultimately based on the EWR Airport Arrival Rate; and IDM plus Required Time of Arrival (RTA), a flight deck tool which allowed some aircraft to meet a controlled time of arrival to the TBFM area more precisely.

Results showed that the checkbox tool was powerful: with the checkbox $\mathrm{ON}$, departure delays decreased and airborne delays increased, as predicted. However, assuming that the cost ratio of a minute of airborne delay to a minute of departure delay is in the range of 1.2 to 3 , as commonly indicated by the literature, checkbox ON and checkbox OFF conditions showed approximately equal total delay costs, i.e., the cost of delays in the air balanced the cost of the delay on the ground. The three scheduling conditions also had approximately equal total delay costs, although a simulation artifact may have reduced the delays in the Baseline condition.

In the debrief following the simulation, the TMCs concluded that the checkbox should be used flexibly depending on the current delay situation, and suggested modifications to the checkbox tool which would help them use it in this way, along with enhanced training. The relatively similar total cost of both checkbox default options in this simulation indicates that this might be a fruitful approach, and replace the necessity to have the checkbox rigidly set to either ON or OFF.

\section{INTRODUCTION}

The introduction of Time-Based Flow Management (TBFM) departure scheduling to high volume airports, such as Newark, LaGuardia, and Atlanta, has significantly improved airborne arrival operations. This is because aircraft can be scheduled directly to their destination runway thresholds prior to departure, taking any delays they might have on the ground and thereby preventing airborne holding and additional fuel use. However, there is a negative consequence to TBFM departure scheduling for aircraft departing from airports that are near high-volume airports (which are called internal departures in this paper). Aircraft from more distant airports, outside the TBFM area, have earlier departure times and frequently take up all the slots on the runway threshold schedule, leaving very limited slots for internal departures. The internal departures then need to wait for a slot to open up on the TBFM timeline, resulting in excessive delays on the ground. Boston and Washington D.C. Metro area airports are examples of internal departure airports in the New York area.

The Federal Aviation Administration (FAA) has attempted to rectify this situation with an enhancement to the TBFM departure scheduling tool which allows Traffic Management Coordinators (TMCs) to make room in an arrival stream by compressing arrival aircraft to their minimum required spacing to create a full slot for a waiting internal departure. This is accomplished via the departure scheduling window in the TBFM software which includes a checkbox called "Delay Scheduled Flights for This Aircraft Only." When this box is checked, space for a departure aircraft in the oncoming arrival stream is automatically made, where possible, by delaying the Scheduled Times of Arrival (STAs) of those airborne flights that are not yet "frozen" (unable to be moved from their reserved slot). As aircraft approach the TBFM freeze horizon, there may be excess spacing, or partial slots, between the Meter Fix (MF) STAs for these airborne flights. The checkbox algorithm will find these partial slots and delay the airborne flights to create a full slot for the internal departure to be scheduled. The en route controllers working with the TBFM metering times see this additional delay on their scopes, and can then delay the impacted aircraft. If the checkbox is OFF, the positions of the arrival aircraft are treated as "givens" and departures are scheduled around both frozen and unfrozen STAs of the airborne flights, typically resulting in significant ground delay for the internal departures. 


\section{A. Background}

A Human-in-the-Loop (HITL) simulation was conducted in January, 2016, which, in addition to comparing checkbox ON vs. checkbox OFF, compared three different methods of delivering aircraft into the TBFM region on their way to Newark Airport (EWR). Reference [1] more completely describes some of these methods and the concept behind them. Basically, the concept was to use the strategic capability within the Traffic Flow Management System (TFMS), to more precisely manage aircraft into a constrained airspace controlled by TBFM.

The three methods were:

1. A Baseline condition of current operations using Miles-in-Trail (MIT) separation,

2. An Integrated Departure Management (IDM) condition in which all departures were given a scheduled time of departure (Estimated Departure Clearance Time or EDCT) consistent with the EWR Airport Arrival Rate.

3. A condition which combined IDM and Required Time of Arrival (RTA) in which pilots of some aircraft were given specific controlled times of arrival into TBFM airspace [2].

The checkbox ON and OFF conditions were apportioned within the three scheduling conditions, as will be described in the Method section.

\section{METHOD}

\section{A. Simulated Airspace}

The airspace that is the focus of this paper is the TBFM Meter Fix region for aircraft going into EWR, as shown in Fig. 1. In this simulation, the MF freeze horizons were closer to EWR in the simulation than the current fielded distanceapproximately $200 \mathrm{~nm}$ instead of $400 \mathrm{~nm}{ }^{1}$ TBFM monitored aircraft for 90 minutes before they crossed the freeze horizon and once they did, it froze their schedules in such a way that all to that airport's arrival rate. This reserved the slots for those aircraft, enabling the center and TRACON controllers to work towards having the aircraft meet the times of those reserved slots. As in the field, if the checkbox was in the "ON" position, the Scheduled Times of Arrivals (STAs) of unfrozen airborne flights that were upstream of the freeze horizon could be given an additional delay so as to create a full slot between arrival aircraft for a departure being scheduled in the TBFM MF region. aircraft destined for EWR could be accommodated according

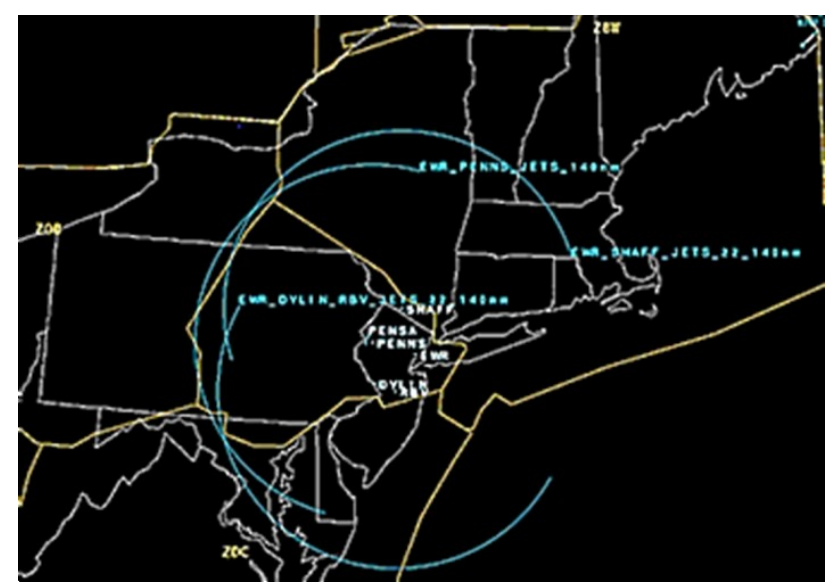

Fig. 1. Arcs are simulated TBFM MF freeze horizons for arrivals going to Newark Airport.

\section{B. Simulation}

The simulation took place at the Airspace Operations Laboratory at NASA Ames Research Center. The TBFM system used in the lab was the FAA fielded version 4.2.3 with NASA modifications and adaptations to fit the new freeze horizon locations. For simplicity, only four meter fixes were used in this evaluation: SHAFF (for traffic from the north), PENNS (for traffic from the west), and DYLIN and RBV (for traffic from the south). These meter fixes are all currently used in the operational TBFM adaptation for EWR. Realistic traffic was simulated, including departure errors based on observed compliances for actual EWR inbound traffic. Two confederate center controllers managed the extended metering traffic to meet the TBFM-generated airborne delays into the MF region.

\section{Participants}

Four participants rotated through one TMC position in the TBFM MF area. The participants were retired SMEs from the following Traffic Management Units (TMUs): New York TRACON (N90), New York Center (ZNY), Boston Center (ZBW), and Fort Worth Center (ZFW).

\section{Experimental Design}

Nine 3-hour simulation runs compared both the checkbox in the default $\mathrm{ON}$ and OFF condition and the three scheduling conditions in a $2 \mathrm{X} 3$ experimental design. As can be seen in Table 1, the checkbox was $\mathrm{ON}$ in six runs and OFF in three runs.

\section{TABLE 1. NUMBER OF RUNS IN EACH CONDITION}

\begin{tabular}{|l|c|c|r|}
\hline $\begin{array}{c}\text { Scheduling } \\
\text { Conditions }\end{array}$ & $\begin{array}{c}\text { Checkbox } \\
\text { ON }\end{array}$ & $\begin{array}{c}\text { Checkbox } \\
\text { OFF }\end{array}$ & \multicolumn{1}{|c|}{ Total } \\
\hline Baseline (MIT) & 2 & 1 & 3 \\
\hline EDCT & 2 & 1 & 3 \\
\hline EDCT + RTA & 2 & 1 & 3 \\
\hline Total & 6 & 3 & 9 \\
\hline
\end{tabular}

\footnotetext{
${ }^{1}$ A new feature of TBFM called extended metering allowed for a second metering point to be placed further out. The extended meter point freeze horizon was placed $400 \mathrm{~nm}$ from EWR to provide additional opportunity to push-back any extra airborne delay for pre-conditioning aircraft into the MF region.
}

There were 142 departure aircraft in the nine runs, with an average of 15.8 internal MF departures in each run. There 
were 1,037 airborne aircraft in the nine runs, with an average of 115.2 aircraft in each run.

\section{E. Tools}

The TBFM tool includes both a timeline graphical user interface (T-GUI) and a planview graphical user interface (P-
GUI), enabling TMCs to monitor traffic and to decide where to schedule internal departures into the arrival streams. Fig. 2 shows the T-GUI and P-GUI of the MF schedule for three flows to EWR. The far left timeline in the TGUI is used for scheduling MF internal departure aircraft.

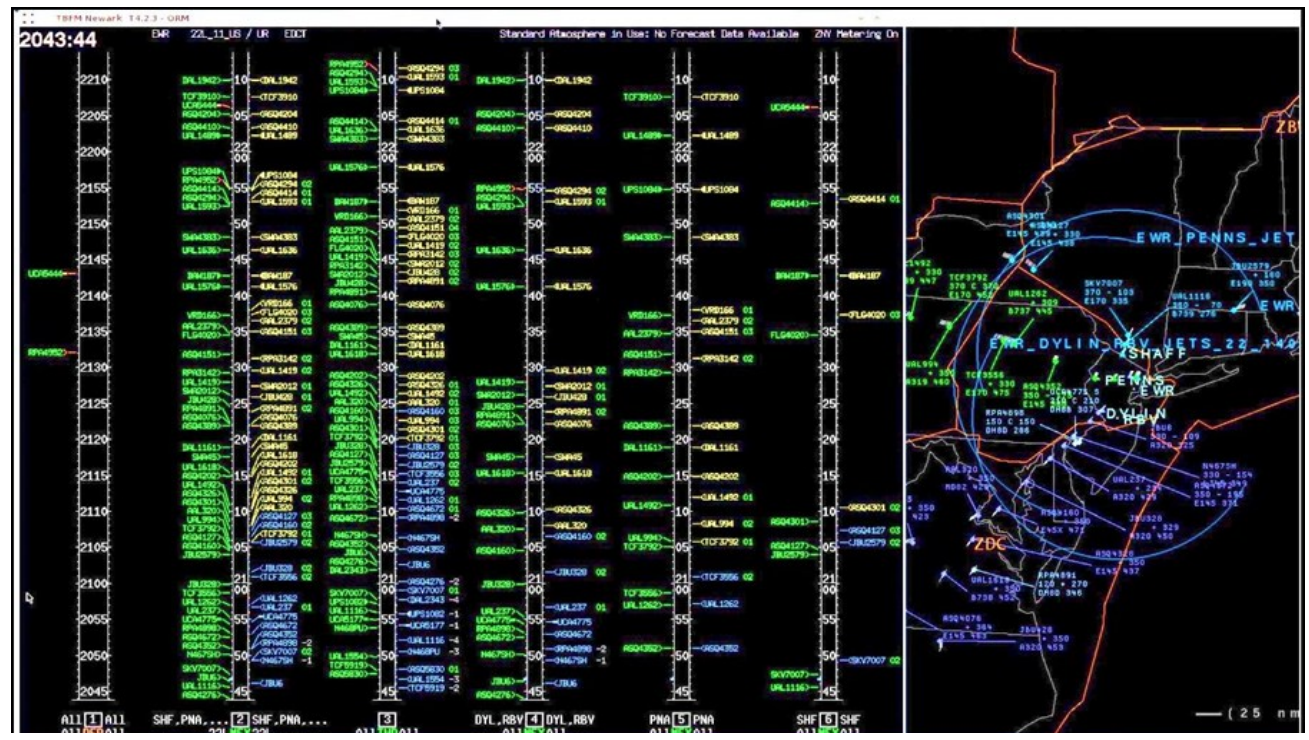

Fig. 2. TBFM T-GUI (left) and P-GUI (right) showing the MF schedule for 3 flows to EWR controlled from NY Center. The far left timeline in the TGUI is used for scheduling MF internal departure aircraft

Fig. 3 shows the "Schedule a Departure" dialogue box, which can be accessed through the F-4 panel in TBFM. The departure scheduler checkbox function "Delay Scheduled Flights for This Aircraft Only" is shown checked, in the ON position, at the bottom of Fig. 3 .

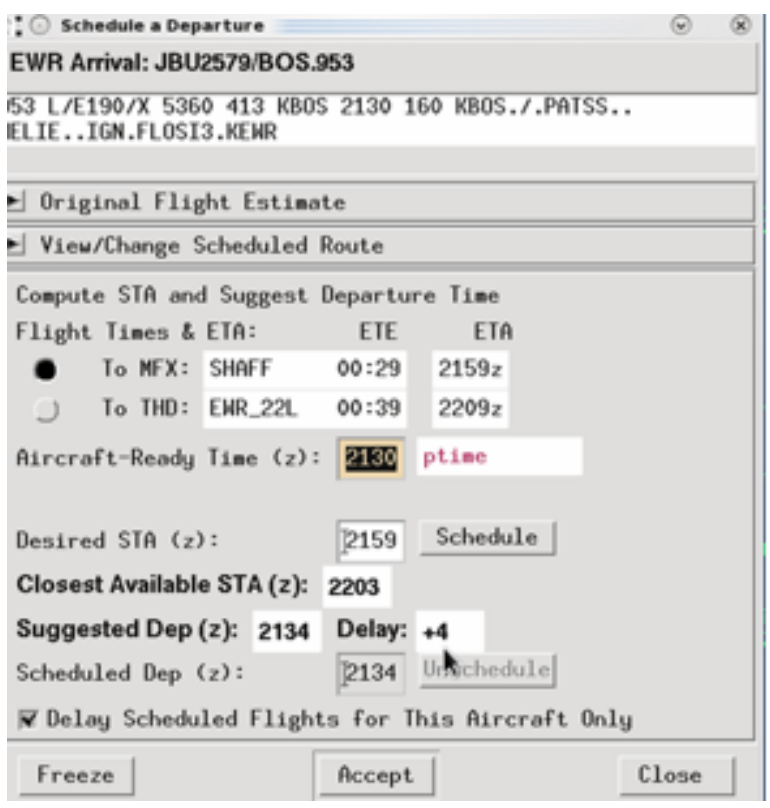

Fig. 3. TBFM scheduling tool with checkbox (at bottom) in the ON position.

\section{F. Procedures}

The main duties of the TMCs were scheduling internal departures, monitoring airport arrival rates, and correcting any aircraft frozen out-of-sequence. To reserve a slot for an internal departure, the TMCs were instructed to freeze the predeparture upon scheduling, as is currently done in the field.

The TMCs were told which default checkbox setting was being used for each run and that if necessary, for operational purposes, they could change the default to ON or OFF for individual aircraft, but that the default setting was preferred. Although the TMCs frequently experimented with both checkbox ON and OFF, in the nine 3-hour runs they actually changed it for only 7 aircraft. They de-activated the checkbox when it was ON for 2 aircraft (both in Baseline conditions), and activated the checkbox from OFF to ON for 5 aircraft (2 in the EDCT-only conditions and 3 in the EDCT plus RTA conditions).

\section{G. Metrics}

Both departure and airborne delays were calculated in seconds for the full simulation run time.

1) Departure Delay Metric: The departure delays consist of both the delays assigned by the TFMS system (EDCTs) and any later delays assigned by the TMC in the TBFM MF area (MF-assigned delays). (Baseline would not have any EDCT delays.)

2) Airborne Delay Metric: The airborne delays were those delays assigned by the TMC in the TBFM MF region. 


\section{RESULTS}

\section{A. Experimental Results}

The results described are in the following sections: 1) Departure Delay, 2) Airborne Delay, 3) Both Departure and Airborne Delay, and 4) Adding Airborne/Departure Delay Cost Ratio.

1) Departure Delay: As shown in Fig. 4, having the checkbox in the default $\mathrm{ON}$ position reduced the average departure delay in the MF area by 7.8 minutes-from 16.2 minutes to 8.4 minutes (Wald Chi Square ${ }^{2}=27.7$, df $1, p=$ $.001)$.

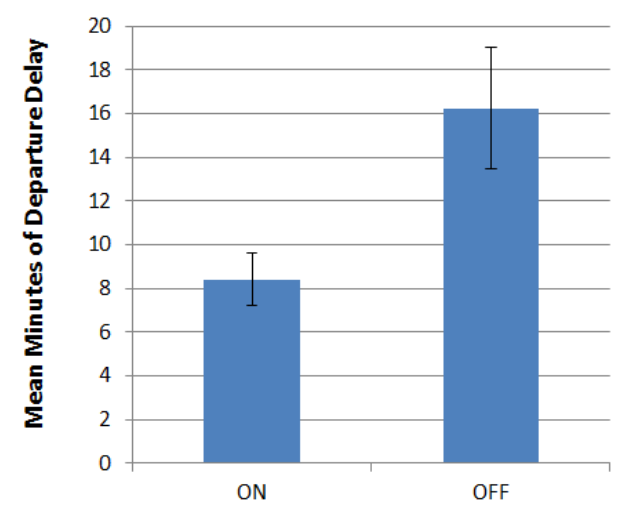

Fig. 4. Average minutes of departure delay with checkbox ON and OFF. Error bars in this and subsequent figures are 95\% Confidence Intervals (CIs), unless otherwise noted.

Fig. 5 shows that with the checkbox ON, the average total departure delays were lower in all conditions, the lowest being in the Baseline checkbox ON condition. (Overall scheduling condition means are 5.2, 14.6, \& 17.8 minutes, Wald Chi Square $=66.5, d f 2, \mathrm{p}=.001$; scheduling condition by checkbox Wald Chi Square $=6, d f 2, p=.048$ ).

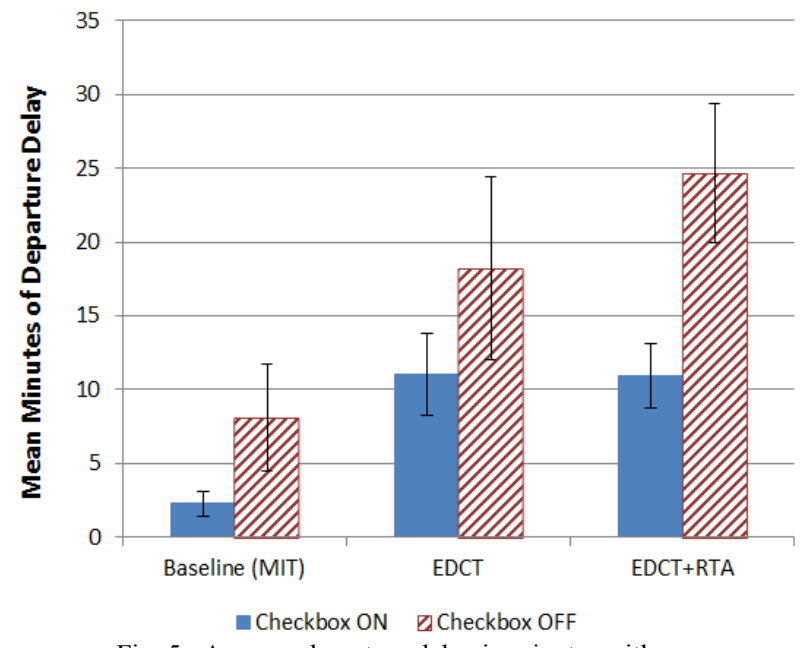

Fig. 5. Average departure delay in minutes with checkbox ON and OFF in the three scheduling conditions.

The average departure delay in the two EDCT scheduling conditions was largely due to the EDCT-assigned delay and not the MF-assigned delay (average of 9.4 minutes vs. 4.8 minutes, paired $t$ test, $t=4.7, d f 92, p<.001)$.

2) Airborne Delay: As shown in Fig. 6, the checkbox in the default $\mathrm{ON}$ position resulted in a slight increase in average airborne delay of 18 seconds per aircraft (means are checkbox ON 89 seconds vs. checkbox OFF 71 seconds, $t(d f$ 999.6, equal variances not assumed) $=2.1, p=.03$ two-tailed, $n=$ 714 checkbox ON and $n=323$ checkbox OFF).

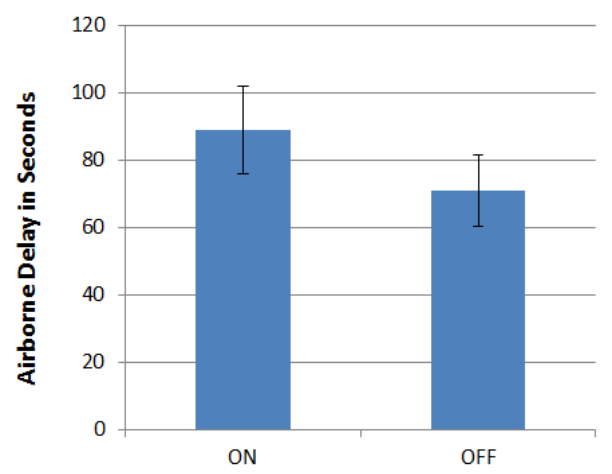

Fig. 6. Average airborne delay in seconds with checkbox ON vs. OFF.

Fig. 7 shows that average airborne delays were reduced in the IDM EDCT scheduling conditions, especially when the checkbox was ON. Hence, more precise delivery to the MF TBFM region reduced average airborne delay within the MF region. (Airborne delay by condition means are 108, 60, 68 seconds, $M S 204,566 F(2,1031)=8.7, p<.001$; checkbox by condition $M S$ 38,569.8, $F(2,1031)=1.5, p=.20$.)

\footnotetext{
2 The departures follow a Poisson distribution [see 3] and are analyzed using the Generalized Linear Model in SPSS for Poisson.
} 


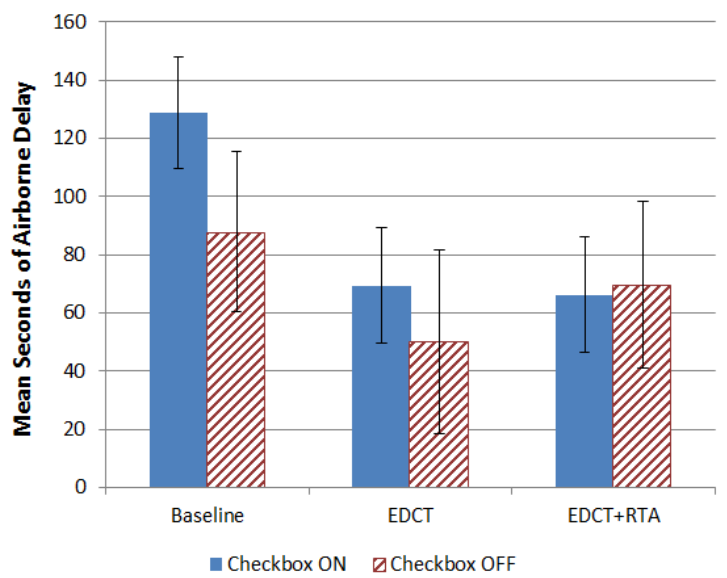

Fig. 7. Average airborne delay in seconds with checkbox ON and OFF in the three scheduling conditions.

3) Both Departure and Airborne Delays: There were many more airborne than departure aircraft in the simulation. The average sums of all aircraft delay per hour of simulation reflect this and are shown in Table 2. In the checkbox ON condition, average sums of airborne delay increased 16.5 minutes and average sums of departure delay decreased 41.7 minutes per hour of simulation.

TABle 2. Average Sums of Airborne and Departure Delay in MINUTES PER HOUR OF SIMULATION WITH CHECKBOX OFF OR ON

\begin{tabular}{|l|r|r|r|}
\hline & $\begin{array}{c}\text { Checkbox } \\
\text { OFF }\end{array}$ & \multicolumn{1}{c|}{$\begin{array}{c}\text { Checkbox } \\
\text { ON }\end{array}$} & Totals \\
\hline $\begin{array}{l}\text { Airborne } \\
\text { delay }\end{array}$ & 42.4 & 58.9 & 101.3 \\
\hline $\begin{array}{l}\text { Departure } \\
\text { delay }\end{array}$ & 84.8 & 43.1 & 127.9 \\
\hline Totals & 127.2 & 102 & 229.2 \\
\hline
\end{tabular}

This is depicted graphically in Fig. 8.

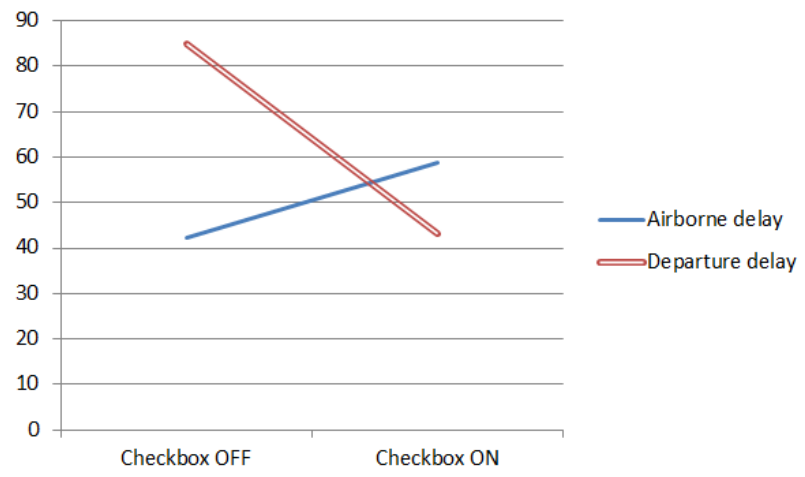

Fig. 8. Average sums of airborne and departure delay in minutes per hour of simulation with checkbox OFF or ON.

4) Adding Airborne/Departure Delay Cost Ratio: A way to judge the impact of these different types of delays is to consider their relative costs. Both departure and airborne delays are costly. The total delay cost of a run, $c$, can be estimated by using

where

$$
c=d+r a
$$

$d=$ sums of departure delay (minutes/hour of simulation)

$a=$ sums of airborne delay (minutes/hour of simulation)

$r=$ ratio of airborne delay cost over departure delay cost.

The average delay sums for checkbox ON and OFF that were originally presented in Table 2 and Fig. 8 , are plotted in Fig. 9. Departure delay is on the $x$ axis and airborne delay is on the $y$ axis, both in minutes per simulation hour. On this graph, we have also plotted a series of lines corresponding to the most common independent estimates of $r$ in the literature. These estimates (see review in [4]), are based on purely economic factors like the cost of fuel, and the values range from 1.2 to 3 [5, 6, 7, 8, see 9 for current values]. All of these lines appear to offer acceptable fits to the data. This is consistent, therefore, with approximately equal total costs for checkbox ON and OFF runs.

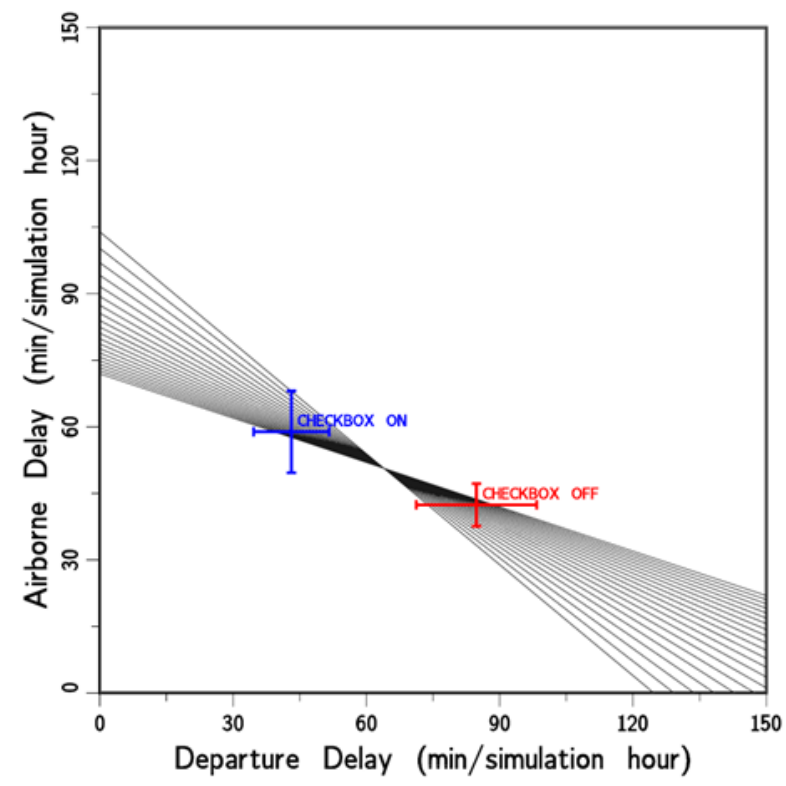

Fig. 9. Airborne and departure delay in average min. per hour of simulation with checkbox ON and OFF. Error bars are standard errors (SEs). Lines are various airborne/departure delay cost ratios from 1.2 to 3 found in the literature. Checkbox $\mathrm{ON}$ and $\mathrm{OFF}$ are therefore about equal in relative cost.

In Fig. 10 similar delays for all nine simulation runs are plotted along with equal cost lines corresponding to $r=2$ (which is near the middle of the independently determined values of the cost ratio). The arrow pointing to the upper right from the origin is perpendicular to the equal cost lines and thus indicates the direction of increasing total costs. 


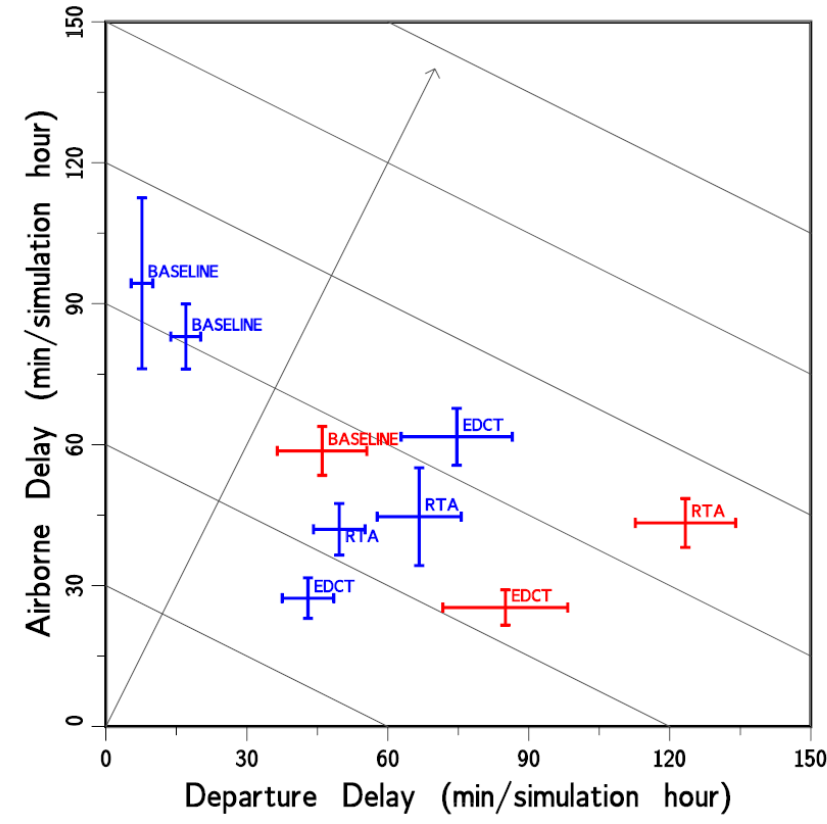

Fig. 10. Airborne by departure delay in minutes per hour of simulation in each of nine runs. Error bars are $S E$ s. Multiple parallel lines show equality of cost if $r=2$ (e.g., 60 minutes of airborne delay equals 120 minutes of departure delay). The line perpendicular to the parallel lines indicates the direction of higher total cost of the runs. Error bars $=S E$ s. Blue indicates checkbox ON condition; red indicates checkbox OFF.

The three runs comprising each scheduling condition are combined in Fig. 11. Although with $r=2$, the EDCT and EDCT+RTA runs appear to be slightly less costly overall than the Baseline runs, they are within the range of equality if the airborne/departure delay cost ratio is between 1.2 and 3 as reported in the literature (see Fig. 9). As the delay cost ratio becomes higher, the IDM conditions would become less costly than the Baseline condition.

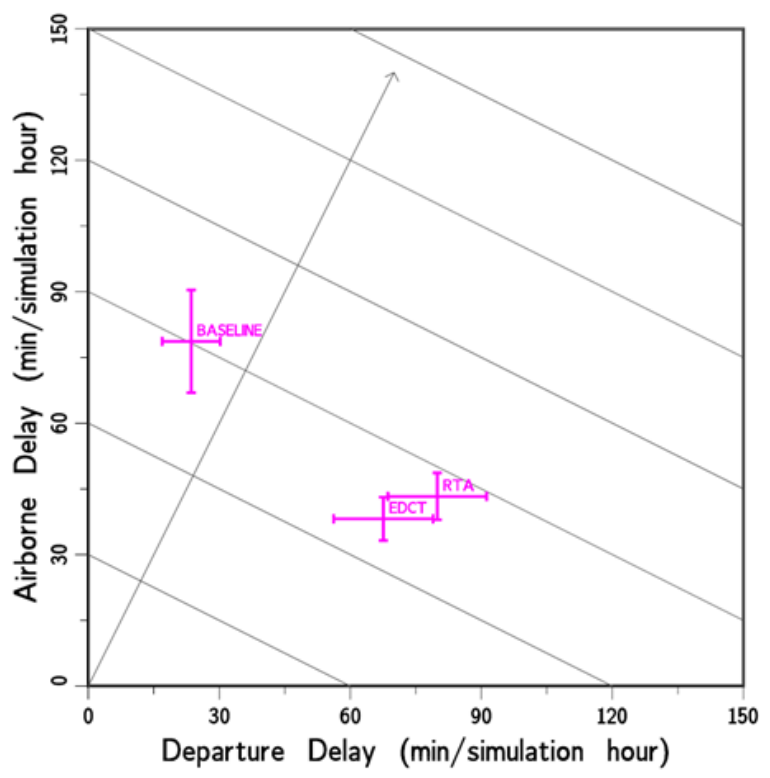

Fig. 11. Relative cost of delays showing approximately equal costs between scheduling conditions. Error bars $=S E$ s.
There is evidence that in the field, both the airborne and departure delays in the Baseline condition would be higher than in the simulation. In all runs in the simulation, the number of airborne aircraft into TBFM was held constant and the TBFM buffer was set at a constant setting (0.0). However, with the particular set of low winds and low final approach speeds in the simulation, TBFM actually allowed a 46-7 rate in the Baseline condition instead of the 44 rate imposed by pre-conditioning the EDCT conditions. Hence in the IDM EDCT conditions, the same number of aircraft were restricted to a lower arrival rate because they were pre-conditioned to do so before entering the TBFM area. If the effective rate had been 44 for the Baseline condition as well, this condition undoubtedly would have had more airborne and departure delay.

\section{B. Participant Assessments}

TMCs responded to a post-run questionnaire and a postsimulation questionnaire and debrief. When asked in the postsimulation questionnaire, "How effective was the checkbox ON position in making space for internal departures," the TMCs rated it an average of 4.5 on a 1 to 5 scale, where 5 represents "Very effective," as shown in Fig. 12.

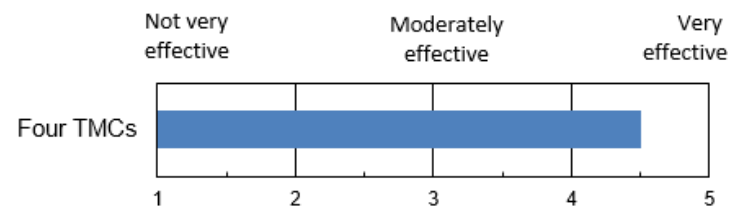

Fig. 12. Ratings of effectiveness of checkbox $\mathrm{ON}$ in making space for internal departures by four TMCs.

In the post-sim questionnaire, the TMCs indicated that the most effective conditions in making space for the internal departures were the EDCT conditions with the checkbox ON, as shown in Fig. 13 (means 4, 4, 3.7, 3.4, 3.4, 3).

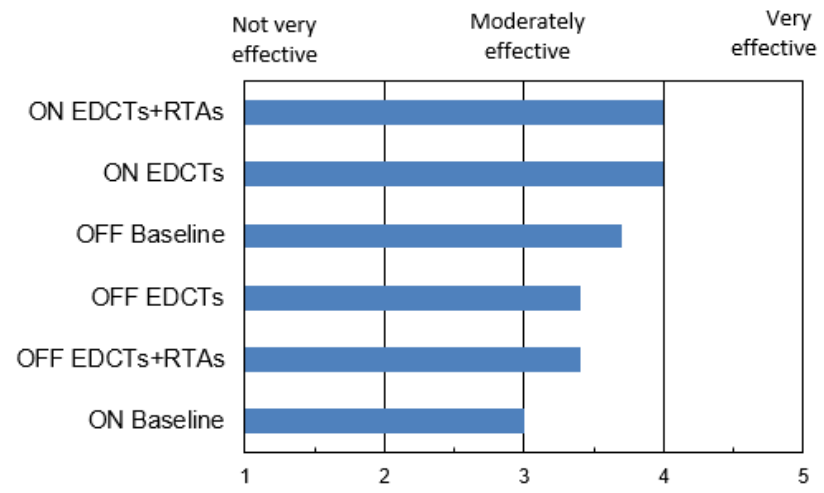

Fig. 13. Ratings by four TMCs of most effective conditions for making space for the internal departures.

When asked in the post-sim questionnaire whether the default checkbox setting be in the "ON" or "OFF" position, the four TMCs recommended that the checkbox be in the default "ON" position. Some of the reasons given were: 
- $\quad$ "You could have a slot that the aircraft could fit in and [if the checkbox were OFF,] it would just schedule the aircraft to the back of the pack."

- " "Able to maximize [departure] slots."

- $\quad$ "Departure delays are typically higher and there is a possibility that the aircraft's impact on the overhead [traffic stream] is not that significant."

When asked about any disadvantages to leaving the checkbox ON, one TMC responded that it would be necessary to monitor the airborne delays to make sure they remained manageable.

However, in the debrief discussion following the simulation, the TMCs all agreed with a comment made by one TMC in the post-sim questionnaire: "Default ON or OFF should not be a constant. Choice should be made based on the actual traffic situations." Also, the TMCs suggested that they be given more information on the effect of having the checkbox ON or OFF. As another TMC wrote, "I would modify the procedure to let you view what would happen with the button on and with the button off." In other words, the TMCs in the debrief recommended that the tool show not only the original departure delay time that is currently visible in the user interface, but the delay time with the checkbox both ON and OFF. Also, when deciding whether it was worthwhile to reduce a departure's delay, the TMCs took into consideration the effects that any additional arrival aircraft delay would have on the en route controllers' workload. In particular, the TMCs weighed two factors: 1) the number of airborne aircraft that would be delayed (preferably less than five aircraft) and 2) the length of the new delays (preferably less than two minutes). The TMCs felt that these numbers could be made more accessible in the user interface and indeed, could be incorporated into a future, more automated decision support tool. Additional training would enable the TMCs to take advantage of this more flexible tool.

\section{DISCUSSION}

In this HITL, whether the TBFM departure scheduling tool checkbox was ON or OFF did not make much difference in terms of total departure and airborne delay cost using the cost ratio values commonly found in the literature. This suggests that there is no reason to rigidly restrict the default. The checkbox tool, which indeed appeared powerful in allocating delay to either departure or airborne aircraft, may be more useful if TMCs use it flexibly, depending on the delay situation on the ground and in the air, as the TMCs in this simulation suggested. The TMCs' specifications of the information needed, such as quick access to airborne and departure delay with the tool ON or OFF, and the number of airborne aircraft affected, would be helpful in enabling the tool to be used this way. Additional training would help the TMCs gain proficiency in this regard.

A more automated tool eventually could assess the departure and airborne delay at any given time and apply the current delay cost ratio to suggest periods of time when the checkbox could remain in an ON or OFF position. This could reduce the time needed for TMCs to monitor airborne and departure delays.

The delay costs of the three types of scheduling were also approximately equal in this simulation. This could be due to the difference in the effective arrival rate into TBFM: 46-47 in Baseline vs. 44 in the IDM conditions. Additional research is therefore needed to address the delay costs of the three types of scheduling.

\section{SUMMARY AND CONCLUSION}

A HITL was conducted in an attempt to simulate different conditions that might reduce departure delays at airports that are near other, higher volume airports. The HITL tested a tool in combination with different scheduling procedures. The tool tested was a TBFM departure scheduling tool-checkbox default $\mathrm{ON}$ vs. default $\mathrm{OFF}$. When $\mathrm{ON}$, this tool can compress airborne aircraft to make space for a departure. The three scheduling conditions were 1) a Baseline condition with Milesin-Trail spacing restrictions, 2) an EDCT condition, where all aircraft were assigned departure times to be consistent with the airport arrival rate at Newark Airport, and 3) an EDCT plus RTA condition which enabled some pilots to more precisely meet their times.

As predicted, the departure delay was reduced with the checkbox in the ON position and arrival delay was increased. The total cost of airborne and departure delay, however, when using common departure to airborne delay cost ratios found in the literature, was about equal in both checkbox ON or OFF conditions.

The TMCs participating in the simulation suggested a flexible approach to the default checkbox ON and OFF option, an approach more sensitive to the variation in departure and airborne delay in a particular situation. The relatively similar total cost of both checkbox default options in this simulation indicates that this might be a fruitful approach, and replace a rigid default setting. The TMCs suggested improvements to the checkbox tool that would make this more flexible approach easier, and quicker to accomplish. Increased training would also help the TMCs take advantage of this approach. Future work in this area could compare the effectiveness of a default OFF checkbox condition with this more adaptive, "Flexible," checkbox condition.

The three scheduling conditions tested in this simulation were also about equally costly when considering airborne and departure delays using current airborne/delay cost ratios found in the literature. This may have been due to a simulation artifact, that is, a slightly higher effective arrival rate in the Baseline condition.

It was shown that these conclusions are dependent on the airborne/departure delay cost ratio. A simple graphic was introduced to clarify the relationship between airborne and departure delay and how the total delay cost varies depending on the different cost ratios. 


\section{ACKNOWLEDGMENT}

The authors are grateful to David Matheson of SPSS IBM Statistics, Chicago, for his expert help on Poisson distribution statistics and integrating $\mathrm{R}$ with SPSS.

\section{REFERENCES}

[1] N.M. Smith, C. Brasil, P.U. Lee, N. Buckley, C Gabriel, C. Mohlenbrink, F. Omar, B. Parke, C. Speridakos, and H.S. Yoo, "Integrated demand management: Coordinating strategic and tactical flow scheduling operations," Proceedings of the $16^{\text {th }}$ AIAA Aviation, Technology, Integration, and Operations (ATIO) Conference, Washington D.C., June, 2016.

[2] H.S. Yoo, N.M. Smith, P.U. Lee, C. Brasil, C. Mohlenbrink, C. Speridakos, O. Faisal, B. Parke, A. Globus, C. Walter, and G. Hodell, "Required Time of Arrival (RTA) as a control mechanism to mitigate uncertainty in arrival traffic demand management," Proceedings of the 35th Digital Avionics Systems Conference (DASC), Sacramento, CA, Sept., 2016.

[3] E. R. Mueller and G. B. Chatterji, "Analysis of aircraft arrival and departure delay characteristics." Proceedings of the AIAA Aviation, Technology, Integration, and Operations (ATIO) Conference. October, 2002.

[4] J. Xiong, "Revealed preference of airlines' behavior under air traffic management initiatives," Dissertation from UC Berkeley, 2010, retrieved on 7/10/16 from http://eprints.cdlib.org/uc/item/7449k7vk

[5] A. Cook, G. Tanner, S. Anderson, "Evaluating the true cost to airlines of one minute of airborne or ground delay," February, 2004. Retrieved on 5/12/2016 from http://www.eurocontrol.int/sites/default/files/content/documents/officialdocuments/facts-and-figures/coda-reports/prc-cost-of-delay-2004.pdf

[6] A. Cook, and G Tanner, "European airline delay cost reference values: Updated and extended values," Version 4.1, December, 2015. Retrieved on 5/12/2016 from http://www.eurocontrol.int/sites/default/files/publication/files/europeanairline-delay-cost-reference-values-final-report-4-1.pdf

[7] A. Q. Kara, J. Ferguson, K. Hoffman, and L. Sherry, "Estimating domestic U.S. airline cost of delay based on European model," International Conference on Research in Air Transportation, (ICRAT 2010), Budapest, Hungary, June, 2010.

[8] Personal communication from Delta Airlines Dispatcher J. Gaanas indicating operational assumption of airborne holding to departure delay of 3 to 1 cost ratio on 6/15/16.

[9] Airlines for America, "Per-minute cost of delays to U.S. Airlines," regularly updated website reflecting changing fuel prices. Retrieved on 5/12/16 from http://airlines.org/data/per-minute-cost-of-delays-to-u-sairlines/ 